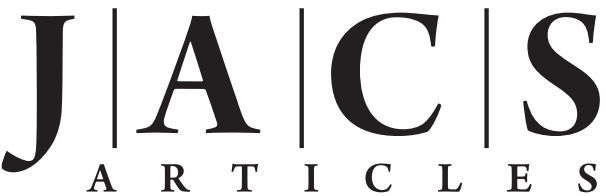

Published on Web 07/26/2010

\title{
Control of the Orientational Order and Nonlinear Optical Response of the "Push-Pull" Chromophore RuPZn via Specific Incorporation into Densely Packed Monolayer Ensembles of an Amphiphilic Four-Helix Bundle Peptide: Characterization of the Peptide-Chromophore Complexes
}

\author{
Venkata Krishnan, ${ }^{\dagger}$ Andrey Tronin, ${ }^{\dagger}$ Joseph Strzalka, ${ }^{\dagger}{ }^{\S} \mathrm{H}$. Christopher Fry, ${ }^{\dagger}$ \\ Michael J. Therien, ${ }^{\ddagger}$ and J. Kent Blasie ${ }^{\star \dagger}$ \\ Departments of Chemistry, University of Pennsylvania, Philadelphia, Pennsylvania 19104, and \\ Duke University, Durham, North Carolina 27708
}

Received February 12, 2010; E-mail: jkblasie@ sas.upenn.edu

\begin{abstract}
Push-pull" chromophores based on extended $\pi$-electron systems have been designed to exhibit exceptionally large molecular hyperpolarizabilities. We have engineered an amphiphilic four-helix bundle peptide to vectorially incorporate such hyperpolarizable chromophores having a metalloporphyrin moiety, with high specificity into the interior core of the bundle. The amphiphilic exterior of the bundle facilitates the formation of densely packed monolayer ensembles of the vectorially oriented peptide-chromophore complexes at the liquid-gas interface. Chemical specificity designed into the ends of the bundle facilitates the subsequent covalent attachment of these monolayer ensembles onto the surface of an inorganic substrate. In this article, we describe the structural characterization of these monolayer ensembles at each stage of their fabrication for one such peptide-chromophore complex designated as AP0-RuPZn. In the accompanying article, we describe the characterization of their macroscopic nonlinear optical properties.
\end{abstract}

\section{Introduction}

Chromophores designed to possess extended $\pi$-electron systems can exhibit exceptionally large molecular hyperpolarizabilities. $^{1-18}$ These chromophores achieve this by maximizing the difference in the electric dipole moments of the ground and excited electronic states while minimizing the HOMO-LUMO band gap. ${ }^{19-23}$ Dipolar or "push-pull" chromophores often exploit a conjugated bridge coupling integral electron donor and acceptor elements. A remarkable class of these hyperpolarizable chromophores features a structural motif in which (porphinato)metal (PM) and metal(II)polypyridyl (M) units are connected via ethyne bridges, ${ }^{8,9,13-15,18}$ these species have been shown to exhibit large hyperpolarizabilities at longer wavelengths relevant to telecommunications applications. ${ }^{8}$ One such chromophore is the linearly conjugated ruthenium(II) [5-(4'ethynyl-(2,2'; $6^{\prime}, 2^{\prime \prime}$-terpyridinyl) $)$-10,20-bis(phenyl)porphinato]zinc(II)-(2, $2^{\prime} ; 6^{\prime}, 2^{\prime \prime}$-terpyridine $)^{2+}$ complex, henceforth designated as RuPZn.

Realization of these large molecular hyperpolarizabilities in potential nonlinear optical device applications, particularly those based on macroscopic second-order effects via $\chi^{(2)}$, typically requires that these chromophores be vectorially oriented in noncentrosymmetric macroscopic ensembles at high number densities. ${ }^{24}$ While interchromophore interactions can degrade the macroscopic nonlinear optical response at very high number

\footnotetext{
University of Pennsylvania.

$\$$ Duke University.

${ }^{\S}$ Current address: X-ray Science Division, Argonne National Laboratory, Argonne, IL 60439.
}

densities, ${ }^{25}$ the response is otherwise usually manifest as a summation of the chromophore's molecular hyperpolarizability, the uppermost limit being a coherent sum, ${ }^{26}$ depending critically on the orientational distribution over optical length scales of the NLO-active species in the ensemble. ${ }^{27}$ Several methods have

(1) Meyers, F.; Marder, S. R.; Pierce, B. M.; Brédas, J. L. J. Am. Chem. Soc. 1994, 116, 10703-10714.

(2) Marder, S. R.; Cheng, L.-T.; Tiemann, B. G.; Friedli, A. C.; BlanchardDesce, M.; Perry, J. W.; Skindhøj, J. Science 1994, 263, 511-514.

(3) LeCours, S. M.; DiMagno, S. G.; Therien, M. J. J. Am. Chem. Soc. 1996, 118, 11854-11864.

(4) LeCours, S. M.; Guan, H. W.; DiMagno, S. G.; Wang, C. H.; Therien, M. J. J. Am. Chem. Soc. 1996, 118, 1497-1503.

(5) Priyadarshy, S.; Therien, M. J.; Beratan, D. N. J. Am. Chem. Soc. 1996, 118, 1504-1510.

(6) Karki, L.; Vance, F. W.; Hupp, J. T.; LeCours, S. M.; Therien, M. J. J. Am. Chem. Soc. 1998, 120, 2606-2611.

(7) Anderson, H. L. Chem. Commun. 1999, 2323-2330.

(8) Uyeda, H. T.; Zhao, Y. X.; Wostyn, K.; Asselberghs, I.; Clays, K.; Persoons, A.; Therien, M. J. J. Am. Chem. Soc. 2002, 124, 1380613813.

(9) Duncan, T. V.; Rubtsov, I. V.; Uyeda, H. T.; Therien, M. J. J. Am. Chem. Soc. 2004, 126, 9474-9475.

(10) Kang, H.; Facchetti, A.; Zhu, P. W.; Jiang, H.; Yang, Y.; Cariati, E.; Righetto, S.; Ugo, R.; Zuccaccia, C.; Macchioni, A.; Stern, C. L.; Liu, Z. F.; Ho, S. T.; Marks, T. J. Angew. Chem., Int. Ed. 2005, 44, 79227925.

(11) Zhang, T. G.; Zhao, Y. X.; Asselberghs, I.; Persoons, A.; Clays, K.; Therien, M. J. J. Am. Chem. Soc. 2005, 127, 9710-9720.

(12) Zhang, T. G.; Zhao, Y. X.; Song, K.; Asselberghs, I.; Persoons, A.; Clays, K.; Therien, M. J. Inorg. Chem. 2006, 45, 9703-9712.

(13) Duncan, T. V.; Ishizuka, T.; Therien, M. J. J. Am. Chem. Soc. 2007, 129, 9691-9703.

(14) Duncan, T. V.; Song, K.; Hung, S. T.; Miloradovic, I.; Nayak, A.; Persoons, A.; Verbiest, T.; Therien, M. J.; Clays, K. Angew. Chem., Int. Ed. 2008, 47, 2978-2981. 
been employed to produce oriented ensembles of hyperpolarizable chromophores. ${ }^{28}$ However, theoretical calculations suggest that the lack of control over the interactions between chromophores in these systems is most likely responsible for their inability to achieve predicted material performance. ${ }^{29,30}$ Achieving homogeneous long-range acentric chromophoric order in a bulk material remains challenging.

In the past decade, artificial proteins have been designed de novo to possess robust $\alpha$-helical bundle motifs. By positioning one or more histidine residues at strategic positions in the sequence forming the helices, they can incorporate both biological and nonbiological chromophores either on their surface or into their core with high specificity, utilizing axial histidyl ligation of a metalloporphyrin moiety. ${ }^{31-35}$ Incorporation of linearly extended chromophores possessing a metal porphyrin component into the core of a designed, amphiphilic $\alpha$-helical bundle peptide can provide several advantages. The interior of the $\alpha$-helical bundle scaffold can be designed to control the position, local dielectric environment via selected residue sidechain interactions, and orientation of the extended conjugation axis of the chromophore within the bundle. Residues on the exterior can be patterned to provide well-defined hydrophilic and hydrophobic domains along the length of the bundle. This amphiphilicity allows the peptide-chromophore complex to be vectorially oriented in macroscopic $2-\mathrm{D}$ ensembles at an interface between polar and nonpolar media, e.g., as in a

(15) Keinan, S.; Therien, M. J.; Beratan, D. N.; Yang, W. J. Phys. Chem. A 2008, 112, 12203-12207.

(16) Reeve, J. E.; Collins, H. A.; Mey, K. D.; Kohl, M. M.; Thorley, K. J.; Paulsen, O.; Clays, K.; Anderson, H. L. J. Am. Chem. Soc. 2009, 131, 2758.

(17) Therien, M. J. Nature 2009, 458, 716.

(18) Hu, X.; Xiao, D.; Keinan, S.; Asselberghs, I.; Therien, M. J.; Clays, K.; Yang, W.; Beratan, D. N. J. Phys. Chem. C 2010, 114, 23492359.

(19) Risser, S. M.; Beratan, D. N.; Mardar, S. R. J. Am. Chem. Soc. 1993, $115,7719-7728$

(20) Prasad, P. N.; Williams, D. J. Introduction to Nonlinear Optical Effects in Molecules and Polymers; Wiley: New York, 1991.

(21) Verbiest, T.; Houbrechts, S.; Kauranen, M.; Clays, K.; Persoons, A. J. Mater. Chem. 1997, 7, 2175-2189.

(22) Wolff, J. J.; Wortmann, R. Adv. Phys. Org. Chem. 1999, 32, 121217.

(23) Dalton, L. R.; Steier, W. H.; Robinson, B. H.; Zhang, C.; Ren, A.; Garner, S.; Chen, A.; Londergan, T.; Irwin, L.; Carlson, B.; Fifield, L.; Phelan, G.; Kincaid, C.; Amend, J.; Jen, A. J. Mater. Chem. 1999, 9, 1905-1920.

(24) Dalton, L. R.; Sullivan, P. A.; Bale, D. H.; Olbricht, B. C. Solid-State Electron. 2007, 51, 1263-1277.

(25) Harper, A. W.; Sun, S.; Dalton, L. R.; Garner, S. M.; Chen, A.; Kalluri, S.; Steier, W. H.; Robinson, B. H. J. Opt. Soc. Am. B 1998, 15, 329337.

(26) Maury, O.; Le Bozec, H. Acc. Chem. Res. 2005, 38, 691-704.

(27) Andrews, D. L.; Allcock, P. In Modern Nonlinear Optics, 2nd ed.; Evans, M. W., Ed.; Wiley: New York, 2001; Vol. 1H, pp 603675.

(28) Burland, D. M.; Miller, R. D.; Walsh, C. A. Chem. Rev. 1994, 94 31.

(29) Dalton, L. R.; Harper, A. W.; Robinson, B. H. Proc. Natl. Acad. Sci. U.S.A. 1997, 94, 4842.

(30) Liakatas, I.; Cai, C.; Bosch, M.; Jager, M.; Bosshard, C.; Gunter, P.; Zhang, C.; Dalton, L. R. Appl. Phys. Lett. 2000, 76, 1368.

(31) Robertson, D. E.; Farid, R. S.; Moser, C. C.; Urbauer, J. L.; Mulholland, S. E.; Pidikiti, R.; Lear, J. D.; Wand, A. J.; DeGrado, W. F.; Dutton, P. L. Nature 1994, 368, 425.

(32) Discher, B. M.; Lear, J. D.; Moser, C. C.; Dutton, P. L. Biophys. J. 2002, 82, 458A.

(33) Discher, B. M.; Koder, R. L.; Moser, C. C.; Dutton, P. L. Curr. Opin. Chem. Biol. 2003, 7, 741.

(34) Ye, S. X.; Strzalka, J. W.; Discher, B. M.; Noy, D.; Zheng, S. Y.; Dutton, P. L.; Blasie, J. K. Langmuir 2004, 20, 5897-5904.

(35) Degrado, W. F.; Summa, C. M.; Pavone, V.; Nastri, F.; Lombardi, A Annu. Rev. Biochem. 1999, 68, 779.
Scheme 1. (a) Amino Acid Sequence (one-letter symbol) for AP0, (b) Chemical Structure of RuPZn Chromophore, and (c) Schematic Representation of APO Peptide and AP0-RuPZn Peptide-Chromophore Complex ${ }^{a}$

(a) APO:

Ac-EIWKLHE.EFLKKFE.ELLKLHE.ERLKKLL.LLALLQL.LLALLQL.GGC-CONH ${ }_{2}$

(b) RuPZn

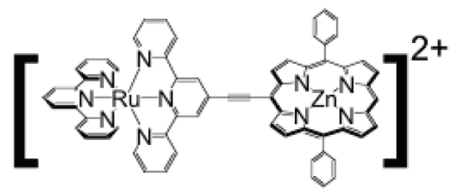

(c)

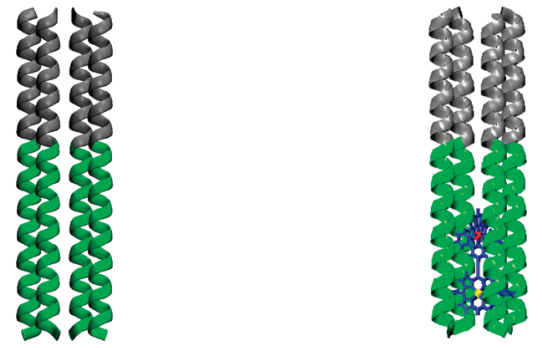

${ }^{a}$ The straight $\alpha$-helices are shown in the ribbon representation: green and grey representing the hydrophilic and hydrophobic domains, respectively, of the amphiphilic four-helix bundle AP0. The RuPZn chromophore is shown in blue, with its zinc and ruthenium atoms shown in yellow and red, respectively. Note that while the sequence contains histidine at positions 6 and 20, only the former has been shown to bind the Zn-porphyrin moiety of the RuPZn chromophore. ${ }^{37}$ That, together with the chromophore's insolubility in polar media, results in the anticipated localization of the chromophore within the nonpolar core of the bundle's hydrophilic domain with the vectorial orientation as shown. Proof of such a unique vectorial orientation of the chromophore within the bundle was recently published. ${ }^{40}$

Langmuir monolayer at the liquid-gas interface, with the longaxis of the bundle perpendicular to the plane of the interface at higher surface pressures. The relatively small cross-sectional dimension of the $\alpha$-helices forming the bundle allows the area per bundle in the plane of the interface to be relatively small, thereby increasing the in-plane density of the peptidechromophore complex to relatively high values while preventing interchromophore contact. In this way the molecular hyperpolarizability of the peptide-chromophore complex may be transformed into a macroscopic nonlinear optical (NLO) material property.

The first de novo designed amphiphilic $\alpha$-helical bundle peptide was designated AP0 (i.e., amphiphilic protein 0). ${ }^{34,36}$ The peptide's primary sequence contains a cysteine residue at its N-terminus (Scheme 1a) that can be utilized to form a dihelical form of the peptide via a covalent disulfide linkage between the N-terminal residues. This peptide was shown to specifically bind both the RuPZn chromophore and a related butadiyne-linked Zn-porphyrin dimer, designated $\mathbf{P Z n E}_{2} \mathbf{P Z n}{ }^{37}$ The properties of these peptide-chromophore complexes in isotropic detergent solutions (including chromophore binding specificities and stoichiometries, helical contents, and thermal stabilities) were extensively described in an earlier publication. ${ }^{37}$ Structural studies of the AP0 peptide itself and of the AP0$\mathbf{P Z n E}_{2} \mathbf{P Z n}$ and AP0-RuPZn peptide-chromophore complexes (at a helix-chromophore mole ratio of 4:1) within Langmuir

(36) Ye, S. X.; Discher, B. M.; Strzalka, J.; Xu, T.; Wu, S. P.; Noy, D.; Kuzmenko, I.; Gog, T.; Therien, M. J.; Dutton, P. L.; Blasie, J. K. Nano Lett. 2005, 5, 1658.

(37) Xu, T.; Wu, S. P.; Miloradovic, I.; Therien, M. J.; Blasie, J. K. Nano Lett. 2006, 6, 2387. 
monolayers at the air-water interface, using X-ray reflectivity, grazing incidence X-ray diffraction, and polarized fluorescence spectroscopy, indicated that the long axis of the chromophore was coincident with the long axis of the amphiphilic four-helix bundle for the emissive $\mathbf{P Z n E} \mathbf{E}_{\mathbf{2}} \mathbf{P Z n}$; likewise, these data showed that the bundle axis was aligned perpendicular to the interface at higher surface pressures for both peptide-chromophore complexes. $^{38}$

However, in these prior works, ${ }^{37,38}$ only the AP0-PZnE $\mathbf{2}_{\mathbf{2}} \mathbf{P Z n}$ complex was extensively investigated. In particular, for the nonemissive RuPZn chromophore in the AP0-RuPZn complex, there was no independent experimental verification of the presence and exact location of the chromophore in the Langmuir monolayer. To this point, we recently developed a method coupling in situ UV-vis absorption spectroscopy and synchrotron X-ray scattering for the structural characterization of Langmuir monolayers. ${ }^{39}$ In this article, we (a) describe the formation of densely packed monolayer ensembles of the amphiphilic four-helix bundle peptide AP0 at the water-gas interface, (b) determine the stoichiometry and firmly establish the mechanism of binding the RuPZn chromophore within the amphiphilic four-helix bundle AP0 vectorially oriented at the water-gas interface, (c) establish the location of the RuPZn chromophore along the length of the bundle consistent with the peptide's design, (d) describe the covalent attachment of these monolayer ensembles of the pure peptide to the smooth planar surfaces of silicon oxide (e.g., as on silicon and fused silica), and (e) the subsequent specific incorporation of the linear "pushpull" chromophore RuPZn into the cores of the peptide bundles attached to these surfaces at stoichiometries as high as two chromophores per bundle. These ensembles have been characterized structurally at each stage of their fabrication, from the Langmuir monolayers of the pure peptide at the water-helium interface by X-ray reflectivity and grazing-incidence X-ray diffraction, to the covalently attached monolayers of the pure peptide at the silicon oxide-helium interface, and ultimately following the incorporation of the chromophore into the peptide at the silicon oxide-helium interface, by X-ray reflectivity enhanced by interferometry. Specimens fabricated in parallel on fused silica were characterized by linear UV-vis absorption spectroscopy to establish the peptide-chromophore stoichiometry. The NLO responses of these latter specimens on fused silica were also investigated in detail and were recently published. ${ }^{40}$

\section{Experimental Section}

2.1. Materials. The amphiphilic peptide AP0 was prepared by solid-phase peptide synthesis using Fmoc chemistry as described in an earlier publication. ${ }^{34}$ Ruthenium(II) [5-(4'-ethynyl- $\left(2,2^{\prime} ; 6^{\prime}, 2^{\prime \prime}\right.$ terpyridinyl))-10,20-bis(phenyl)porphinato]zinc(II)- $\left(2,2^{\prime} ; 6^{\prime}, 2^{\prime \prime}\right.$ terpyridine $)^{2+}$ bis-hexafluoro-phophasphate (RuPZn) was synthesized as described previously. ${ }^{8}$ The amino acid sequence of AP0, the molecular structure of RuPZn, and a schematic representation of AP0 peptide and AP0-RuPZn peptide-chromophore complex are shown in Scheme 1 . The detergent $n$-octyl- $\beta$-D-glucopyranoside (OG) was purchased from Anatrace (Maumee, OH). 3-Aminopropyldimethylethoxysilane was bought from Gelest Inc. (Morrisville, PA) while the linker succinimidyl 4-( $N$-maleidomethyl)cyclohexane-

(38) Strzalka, J.; Xu, T.; Tronin, A.; Wu, S. P.; Miloradovic, I.; Kuzmenko, I.; Gog, T.; Therien, M. J.; Blasie, J. K. Nano Lett. 2006, 6, 2395.

(39) Tronin, A.; Strzalka, J.; Krishnan, V.; Fry, H. C.; Therien, M. J.; Kuzmenko, I.; Blasie, J. K. Rev. Sci. Instrum. 2009, 80, 033102-1.

(40) Gonella, G.; Dai, H.-L.; Fry, H. C.; Therien, M. J.; Krishnan, V.; Tronin, A.; Blasie, J. K. J. Am. Chem. Soc. 2010, 132, 9693-9700. 1-carboxylate (SMCC) was obtained from Fisher Scientific (Springfield, NJ). All other solvents and reagents were also obtained from Fisher Scientific (Springfield, NJ). All purchased chemicals were used without further purification. The fused silica slides (UV grade; $25 \mathrm{~mm} \times 70 \mathrm{~mm}$ ) were bought from Esco Products (Oak Ridge, $\mathrm{NJ}$ ) and the $3 \mathrm{in}$. diameter silicon wafers (n-type Si:P, $600 \mu \mathrm{m}$ thick) were purchased from El-Cat Inc. (Waldwick, NJ). In order to increase the sensitivity and spatial resolution of the X-ray reflectivity technique by employing the interferometric approach, multilayers composed of $50 \AA \mathrm{Si} / 20 \AA \mathrm{Ni} / 20 \AA \mathrm{Si}$ were deposited onto the silicon wafers. This fabrication was performed in the deposition laboratory at the Advanced Photon Source (APS) at Argonne National Laboratory (ANL) (Argonne, IL).

2.2. Langmuir Monolayers at Water-Gas Interface. The Langmuir trough used for the experiments was fabricated from a copper block, coated with Teflon, and manufactured by R\&K, Germany. Potassium phosphate solution $(1 \mathrm{mM})$ at $\mathrm{pH} 8.0$ was used as the aqueous subphase. The temperature of the subphase was maintained constant at $20{ }^{\circ} \mathrm{C}$ using a cooled water circulation in the copper block during the experiment. The AP0 peptide and AP0-RuPZn peptide-chromophore complex, typically having a concentration of about $100 \mu \mathrm{M}$, were spread from detergent solutions to form Langmuir monolayers on the aqueous subphase contained in the Langmuir trough. The detergent solution was prepared by dissolving $1.28 \%$ (mass percent) of $n$-octyl- $\beta$-Dglucopyranoside (OG) in $\mathrm{pH} 8.0$ potassium phosphate buffer. The use of detergent is required to solubilize the amphiphilic four-helix bundle peptides in the buffer solution and also to avoid aggregation/ precipitation of RuPZn chromophore. Experiments were performed with AP0-RuPZn at mole ratios of 4:1, 3:1, 2:1, and 1:1 helices per RuPZn, in the spreading solution, as well as with the AP0 peptide. The as-spread monolayers were equilibrated for about 15 min and then compressed at a rate of approximately $20 \AA^{2}$ per helix per min (i.e., compression from $500 \AA^{2}$ per helix to $100 \AA^{2}$ per helix occurred over $20 \mathrm{~min}$ ). The surface pressure was monitored using a Wilhelmy plate as film balance and the pressure-area isotherms (data not shown) were recorded for both AP0 and all AP0-RuPZn monolayers. The process of compression orients the amphiphilic peptides with the bundle long axis perpendicular to the water-gas interface at high surface pressures. X-ray reflectivity (XR) and grazing incidence X-ray diffraction (GIXD) data were collected on the compressed monolayers having a surface area of $100 \AA^{2}$ per $\alpha$-helix. In combination with X-ray measurements, optical absorption spectra were also recorded in situ on the same monolayer in order to quantify the actual mole ratio (helix: chromophore) in the Langmuir monolayer, as this could be different from that in the spreading solution due to the possible loss of both the peptide and chromophore with the detergent into the subphase upon compression.

A portable UV-vis spectrophotometer (SD 2000, Ocean Optics, Dunedin, FL) utilizing a deuterium/halogen lamp (Model DH-2000, Mikropack $\mathrm{GmbH}$, Germany) coupled with fiber optics to a multipass sensor (utilizing six passes through the monolayer) was used to collect the UV-vis spectra of the Langmuir monolayers under in situ conditions. The detailed description of the instrumentation and data collection has been described in an earlier publication. ${ }^{39}$

The XR and GIXD measurements on the Langmuir monolayers were performed at the liquid surface scattering instrument on the undulator beamline at sector 9 of Advanced Photon Source (APS) at Argonne National Laboratory (ANL), Argonne, IL. The Langmuir trough, enclosed within a gas-tight canister, was circulated with humid He gas in order to reduce the X-ray background scattering arising from air and also to minimize subphase evaporation. The measurements were performed with a X-ray energy of $13474 \mathrm{eV}$ with the storage ring operating at $7 \mathrm{GeV}$ and a beam current of about $100 \mathrm{~mA}$. For the XR measurements, the incident beam slits were set to collect the full horizontal width and vertically to limit the footprint on the liquid surface. A Bicron scintillation detector 
measured the scattered beam intensity, while a He-filled ion chamber before the sample measured the incident beam intensity. The specularly reflected beam from the liquid surface was measured at an angle $\beta$ with respect to the liquid surface in the vertical scattering plane at $2 \theta_{x y}=0^{\circ}$, for $\alpha=\beta$, where $\alpha$ is the angle which the incident beam makes with the liquid surface. Scattered beam slits were set to accept the full specularly reflected beam. Offspecular background was measured at $\alpha=\beta$ with $2 \theta_{x y}= \pm 0.3^{\circ}$. The difference (specular minus off-specular background) provides the reflectivity $R\left(q_{z}\right)$ for photon momentum transfer $q_{z}$ perpendicular to the liquid surface, where $q_{z}=(4 \pi / \lambda) \sin \alpha$. For the GIXD experiments, a Pilatus $100 \mathrm{~K}$ pixel-array detector (Dectris, Switzerland), mounted on the sample- $2 \theta$ arm of the instrument at a specimen-detector-distance of about $30 \mathrm{~cm}$, was used to measure the diffraction pattern, and the measurements were performed at a fixed angle of incidence below the critical angle for the aqueous subphase and $\beta=0^{\circ}$. The specular reflectivity data $\mathrm{I}\left(q_{z}, q_{x y}=0\right.$ $\AA^{-1}$ ) was blocked with a single-crystal tungsten beamstop specially etched to reduce parasitic scattering, allowing the acquisition of the GIXD data $I\left(q_{x y}>0 \AA^{-1}, q_{z}\right)$ in 40 s exposures. Comparison of subsequent time frames showed no evolution of the data indicative of the absence of radiation damage to the peptide. An analytic function that best describes the GIXD data from the subphase was used for scattering background subtraction.

2.3. Monolayers on Solid Substrates. The silicon wafers with multilayer structure were cut into pieces of $20 \mathrm{~mm} \times 10 \mathrm{~mm}$ dimension suitable for X-ray experiments while the fused silica slides were cut into pieces of $25 \mathrm{~mm} \times 35 \mathrm{~mm}$ dimension suitable for linear UV-vis absorption spectroscopy and NLO measurements. Both the silicon wafer slides and fused silica slides were treated together, from cleaning through monolayer deposition. The slides were cleaned using methanol, chloroform, and acetone through sonication in each of these solvents for $10 \mathrm{~min}$. Later the slides were alkylated with 3-aminopropyldimethylethoxysilane under an Ar atmosphere by immersing the substrates in a 5\% silane solution in toluene for $1 \mathrm{~h}$. The slides were rinsed with toluene and sonicated in this solvent for $10 \mathrm{~min}$. Subsequently the slides were cured under Ar at $67{ }^{\circ} \mathrm{C}$ for $1 \mathrm{~h}$. For linker attachment, the slides were placed in $1 \mathrm{mM}$ SMCC solution in anhydrous methanol for $1 \mathrm{~h}$ and then rinsed with methanol, sonicated for $10 \mathrm{~min}$, and dried using a jet of Ar gas.

The Langmuir monolayer of AP0 peptide was formed at water-air interface as described in the previous section, but in this case a commercial Langmuir trough (Lauda, Lauda-Koenigshofen, Germany) was used. The monolayer was compressed to a surface area of $100 \AA^{2}$ per $\alpha$-helix and later deposited onto the previously treated silicon wafer and fused silica slides by a modified Langmuir-Schaefer (LS) deposition. The lower alkylated surface of the horizontal slide was placed in contact with the upper surface of the peptide monolayer. The slides were left in contact overnight to facilitate the covalent attachment between the SMCC linker and the cysteine residue at the upper end of the peptide's helices. The next day, the substrates with the attached AP0 monolayer were washed several times with flowing ultrapure water (Millipore, Billerica, MA) in order to remove nonspecifically adsorbed peptide molecules. The slides were dried using a jet of Ar gas and incubated with $38 \mu \mathrm{M}$ RuPZn solution (in $1.28 \%$ OG pH 8.0 potassium phosphate buffer) at $4{ }^{\circ} \mathrm{C}$ overnight to achieve axial histidyl ligation of RuPZn chromophore to AP0 peptide in order to form AP0RuPZn monolayer. Upon removal from the incubation medium, the substrates were once again washed several times with flowing ultrapure water to remove nonspecifically adsorbed RuPZn molecules. The substrates were stored under $\mathrm{Ar}$ at $4{ }^{\circ} \mathrm{C}$ prior to experimental measurements. These covalently attached single monolayer specimens prepared in parallel should be nearly identical, as both substrates (silicon wafer and fused silica) possess a silicon oxide surface and the same surface functionalization has been performed on both kinds of substrates prior to the monolayer deposition.
The linear UV-vis absorption spectroscopy data were collected at room temperature from the covalent attached monolayer films on fused silica substrates using a Lambda $650 \mathrm{UV}$-vis spectrometer (PerkinElmer, Waltham, MA). The X-ray reflectivity (XR) data for the covalently attached monolayer films on silicon substrates having a multilayer structure were collected using a triple-axis diffractometer with an Enraf-Nonius F-591 rotating-anode X-ray source and a scintillation detector. A curved LiF crystal was utilized to select and collimate the $\mathrm{Cu}-\mathrm{K} \alpha_{1}$ spectral line. Further collimation was achieved by the slits before and after the specimen chamber, with the resolution determined by the analyzer crystal before the scintillation detector. Reflectivity data were recorded over the range of photon momentum transfer $q_{z}$ from 0.01 to $0.62 \AA^{-1}$. At lower incident angles, the X-ray beam was attenuated by nickel foils inserted upstream from the specimen. The specimen chamber with Kapton windows was maintained at $24{ }^{\circ} \mathrm{C}$. A LI-610 dewpoint generator (LI-COR, Lincoln, NE) was additionally fitted to maintain the relative humidity inside the chamber in excess of $96 \%$ in helium.

2.4. Data Analysis. The surface density of the RuPZn chromophore in the Langmuir monolayers and in the covalently attached monolayer films was calculated from the linear UV-vis absorption spectra utilizing the extinction coefficient of the RuPZn chromophore $^{8}\left(\varepsilon_{653 \mathrm{~nm}}=51000 \mathrm{M}^{-1} \mathrm{~cm}^{-1}\right)$. The raw X-ray reflectivity data from both Langmuir monolayers and the covalently attached monolayer films were first reduced using C-Plot (Certified Scientific Software, Cambridge, MA); this procedure included normalization of the reflectivity data by the Fresnel function to eliminate the effects due to dynamical scattering from a single ideal interface, leaving the kinematical scattering due to the presence of either the peptide monolayer itself or the multilayer reference structure with the attached peptide monolayer at the interface, respectively. Subsequent analysis was performed using the Distorted Wave Born Approximation and the so-called box-refinement algorithm, implemented via Mathematica 4.2 (Wolfram Research, Champaign, IL), for solving the phase problem and obtaining the electron-density profile that fully accounts for the observed reflectivity data. This approach was thoroughly described in a prior publication ${ }^{41}$ for Langmuir monolayers and in publications ${ }^{42,43}$ for monolayer films on multilayer reference structures. The raw GIXD data were first reduced using the Datasqueeze 2.1.4 program package (Datasqueeze Software, Wayne, PA), and subsequent analysis was performed with Mathematica 4.2 using analytical expressions as described in an earlier publication. ${ }^{44}$

\section{Results and Discussion}

3.1. In Situ UV-vis Spectra of Langmuir Monolayers at the Water-Gas Interface. In situ UV-vis absorbance spectroscopy was used (a) to check whether the histidine residue of the peptide forms an axial ligand with the $\mathrm{Zn}$ atom in the porphyrin moiety of RuPZn and (b) to evaluate the in-plane density of the so-bound RuPZn chromophore in the Langmuir monolayers of peptide-chromophore complexes, i.e., to determine the actual helix to chromophore mole ratio in the Langmuir monolayers at the water-gas interface. As a representative case, the UV-vis absorption spectrum of AP0-RuPZn in a compressed Langmuir monolayer, whose mole ratio in the spreading solution was 4:1, is shown in Figure 1, along with its spectrum in solution before spreading, and that of the RuPZn chromophore in DMSO. The monolayer spectrum clearly shows all

(41) Blasie, J. K.; Zheng, S.; Strzalka, J. Phys. Rev. B 2003, 67, 224201224208.

(42) Krishnan, V.; Strzalka, J.; Liu, J.; Liu, C.; Kuzmenko, I.; Gog, T.; Blasie, J. K. Phys. Rev. E 2010, 81, 021604.

(43) Kneller, L. R.; Edwards, A. M.; Nordgren, C. E.; Blasie, J. K.; Berk, N. F.; Krueger, S.; Majkrzak, C. F. Biophys. J. 2001, 80, 2248.

(44) Churbanova, I. Y.; Tronin, A.; Strzalka, J.; Gog, T.; Kuzmenko, I.; Johansson, J. S.; Blasie, J. K. Biophys. J. 2006, 90, 3255-3266. 


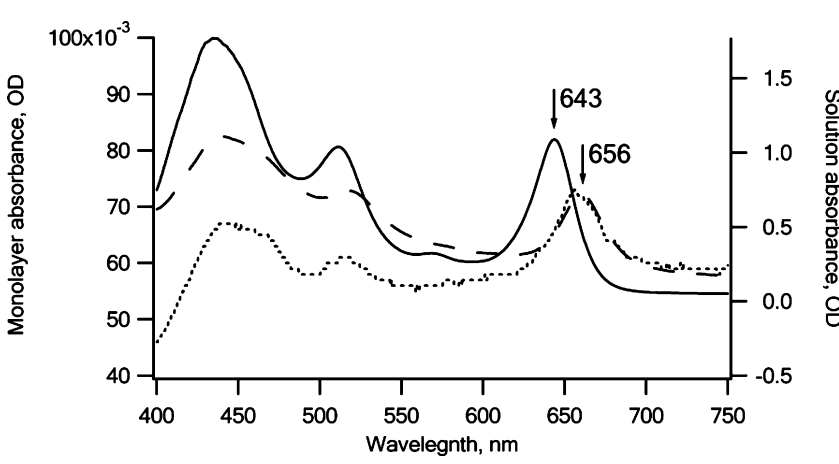

Figure 1. Comparison of absorbance spectrum of RuPZn itself in DMSO (solid; right-side ordinate scale), incorporated into the four-helix bundle peptide AP0 at mole ratio 4 helices: 1 chromophore, in the spreading solution (dashed; right-side ordinate scale), and within a Langmuir monolayer (dotted; left-side ordinate scale). See text for experimental details.

three characteristic bands of RuPZn chromophore: the Soretderived band at $443 \mathrm{~nm}$, a band at $515 \mathrm{~nm}$ featuring substantial metal-ligand charge transfer character, and a $Q_{x}$-derived manifold centered at $656 \mathrm{~nm} .{ }^{8}$ The position of the $\operatorname{RuPZn} Q_{x^{-}}$ derived absorption maximum is sensitive to the ligation state of its (porphinato)zinc unit, and the local dielectric environment, due to the strong mixing of porphyrin $\pi-\pi^{*}$ - and metal polypyridyl-based charge-resonance absorption oscillator strength in this manifold of states. In DMSO solvent, this absorption manifold is centered at $643 \mathrm{~nm}$. Upon binding within the low dielectric interior of the $\alpha$-helical bundle and axial histidyl ligation, the RuPZn $Q_{x}$-derived absorption maximum red-shifts to $656 \mathrm{~nm}$. The position of this RuPZn absorption manifold in the peptide monolayer is the same as for the AP0-RuPZn solution before spreading, demonstrating the preservation of histidyl ligation of the porphyrin-coordinated $\mathrm{Zn}$ ion in the monolayer. This result is nontrivial, due to the modest metal-histidine bond strength and the fact that chromophore binding is expected to be heavily dependent upon the actual conformation of the peptide. The conformation has been demonstrated to be different in detergent solution and in the monolayer, where the tetrahelix bundle dissociates into dihelices at low surface pressures when initially spread at the water-gas interface. The observation of the chromophore being bound to the peptide in the monolayer also agrees with the fact that no appreciable RuPZn absorbance was recorded in the aqueous subphase, on either side of the moving barrier. Calculation of the area per chromophore from the monolayer absorbance provides the value $500 \AA^{2}$ when the monolayer is compressed to an area of $100 \AA^{2}$ per $\alpha$-helix. This indicates that the actual mole ratio is 5:1 in the Langmuir monolayer, although the mole ratio was $4: 1$ in the spreading solution. Similarly, the actual mole ratio of peptide helix to chromophore in the Langmuir monolayer for the other AP0-RuPZn samples were 5:1, 4:1, and 2:1, wherein the mole ratio in the spreading solution was 3:1, 2:1, and 1:1, respectively. We note that for all the AP0RuPZn samples, the $Q_{x}$-derived absorption maximum was at $656 \mathrm{~nm}$, indicating that the chromophore retained its axial ligation to the histidine residue of the peptide, even when spread at the interface and subsequently compressed in the monolayer. This was not the case at other detergent concentrations, so in this sense we determined that spreading solutions with $1.28 \%$ OG were optimal. The actual mole ratios determined using this in situ UV-vis spectroscopy will be used in all further discussions irrespective of the mole ratio of the precursor spreading solution.
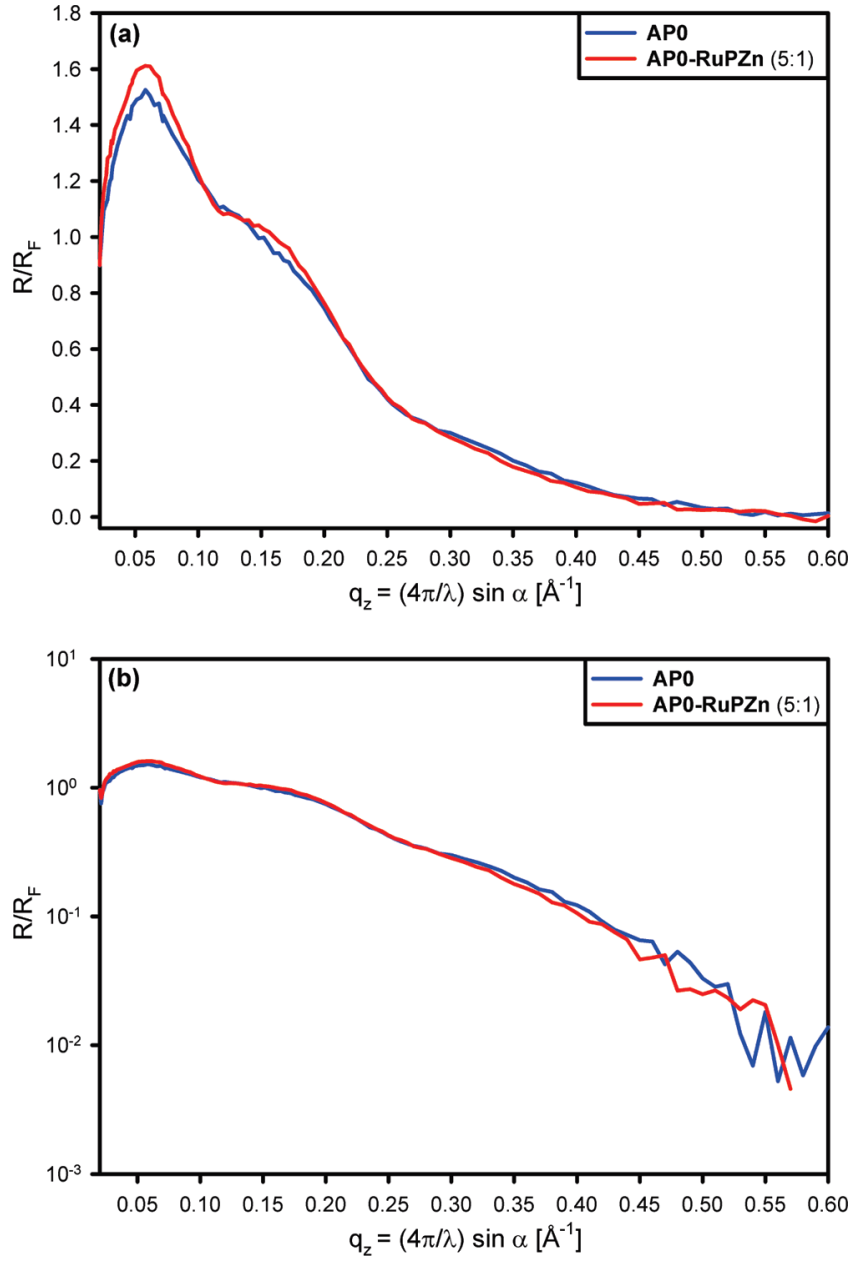

Figure 2. Fresnel-normalized X-ray reflectivity data for compressed Langmuir monolayers of AP0 and AP0-RuPZn (5:1) ((a) linear ordinate scale and (b) log ordinate scale).

3.2. X-ray Reflectivity Studies of Langmuir Monolayers at the Water-Gas Interface. As a representative example, the Fresnel normalized X-ray reflectivity data, $R\left(Q_{z}\right) / R_{\mathrm{F}}\left(Q_{z}\right)$, for $\mathbf{A P 0}$ and AP0-RuPZn (5:1) obtained for surface area of $100 \AA^{2}$ per $\alpha$-helix is shown in Figure 2. The surface area of $100 \AA^{2}$ per $\alpha$-helix corresponds to a surface pressure of about $45 \mathrm{mN} \mathrm{m}^{-1}$ for AP0 and about $50 \mathrm{mN} \mathrm{m}^{-1}$ for AP0-RuPZn (5:1). As mentioned earlier, at these high surface pressures, the $\alpha$-helical bundles are oriented with the bundle long axis perpendicular to the plane of the water-helium interface. The Fresnel normalized reflectivity plot shows a maximum around $0.06 \AA^{-1}$ and a shoulder around $0.15 \AA^{-1}$, features indicative of this orientation of the peptide and peptide-chromophore complex at the water-gas interface. ${ }^{21}$

Fourier transformation of the Fresnel normalized data provides the autocorrelation of the gradient of the electron density profile of the monolayer, also known as the Patterson function. Further analysis, utilizing the profile extent of the gradient profile provided by the Patterson function as a constraint in the boxrefinement procedure, ${ }^{41}$ provided the gradient of the electron density profile of the monolayers. Finally, the electron density profiles themselves for the AP0 and AP0-RuPZn (5:1) monolayers shown in Figure 3 were obtained by numerical integration of the respective gradient profiles. The peptide-gas interface is at $0 \AA$ and the peptide-water interface is at $-60 \AA$ in these profiles. Thus, both the AP0 and AP0-RuPZn (5:1) profiles 


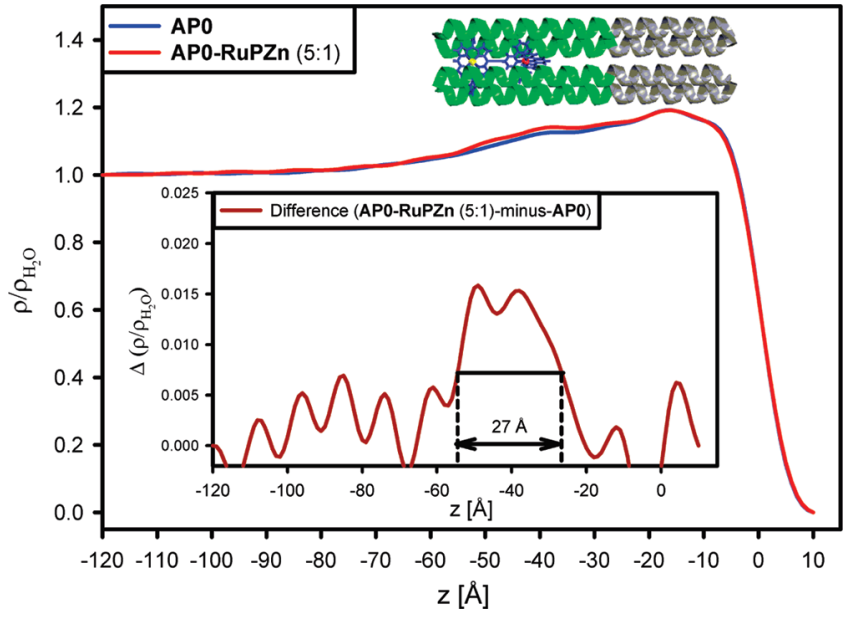

Figure 3. Electron density profiles for compressed Langmuir monolayers of AP0 and AP0-RuPZn (5:1). Schematic representation of AP0-RuPZn is shown above, approximately to scale. Inset: Difference electron density between AP0-RuPZn (5:1) and AP0 monolayers, shown on a magnified ordinate scale.

extend about $60 \AA$ in length perpendicular to the monolayer plane. Overall, each helix of AP0 has 42 residues, which provides a length of $\sim 63 \AA$ for a $\alpha$-helix at $1.5 \AA$ per residue along the long axis. Hence, the experimentally obtained value is in good agreement with the value expected for this peptide.

The electron density profiles of AP0 and AP0-RuPZn (5:1) are qualitatively similar with the same general shape, revealing that the $\alpha$-helix bundles are not substantially distorted due to the incorporation of the RuPZn chromophore. The inset in Figure 3 shows the difference in the electron density profiles, or difference profile, between AP0-RuPZn (5:1) and AP0. Importantly, this difference profile is reproducible, as the reflectivity data for both $\mathbf{A P 0}$ and $\mathbf{A P 0}$-RuPZn are reproducible to within their respective counting statistics, and both scan-toscan on the same monolayer and from monolayer-to-monolayer, ${ }^{45}$ the difference in the reflectivity data between AP0 and AP0-RuPZn exceed these statistics. The difference profile indicates that a significant perturbation of the monolayer profile occurs over the region between $-26 \AA$ and $-53 \AA$ upon incorporation of the RuPZn chromophore. The amplitude of the features characterizing this perturbation are approximately six times the uncertainty level of $\pm 0.0025 \mathrm{e}^{-} \AA^{-3}$, the latter based on the amplitude of the minimum wavelength fluctuations present throughout the difference profile. The identification of significant features in such difference profiles based on their uncertainty levels has been thoroughly discussed in a prior

(45) With regard to monolayer-to-monolayer reproducibility, for the AP0RuPZn peptide-chromophore complex, in situ determination of the peptide helix to chromophore mole ratio was an absolute necessity. Thus, while a particular helix/chromophore mole ratio could not be reproduced exactly monolayer-to-monolayer, systematic differences were obtained with increasing chromophore content of the monolayer. For example, the significant features in the difference profile for AP0RuPZn (5:1)-minus-AP0, shown in the Figure 3 inset and described in the text, occur within the region of the profile containing the hydrophilic domain of the bundle (i.e., $-60 \AA<z<-20 \AA$ ) with an FWHM of $\sim 27 \AA$ and an amplitude of about 6 times the uncertainty level. These features are reproduced in the difference profile for AP0RuPZn (4:1)-minus-AP0-RuPZn (5:1), occurring within the same region of the monolayer profile with an FWHM of $\sim 28 \AA$ and an appropriately smaller amplitude of about 3 times the uncertainty level, thereby effectively establishing their reproducibility in this manner. publication. ${ }^{46}$ The full-width at half-maximum (fwhm) of the features characterizing this perturbation is $\sim 27 \AA$, comparable to the expected length of $24 \AA$ for the RuPZn chromophore. Both the position and width of these features are consistent with the expected localization of the RuPZn chromophore along the length of the peptide bundle, as shown in the schematic representation of the AP0-RuPZn peptide-chromophore complex in Scheme 1. We note that this difference profile represents the total structural perturbation of the peptide bundle within the Langmuir monolayer upon chromphore incorporation that includes changes in the local conformation of the bundle and its hydration as well the chromophore's additional electron density. The amplitude of the features characterizing this perturbation is relatively small here because the helix to chromophore stoichiometry in the monolayer is only 5:1 and only about $60 \%$ of the election density in this region arises from the peptide-chromophore complex itself, the remainder being from water.

The Fresnel normalized X-ray reflectivity data and corresponding electron density profiles for AP0-RuPZn in mole ratios of 4:1 and 2:1 are shown in Figure S1 (Supporting Information). For mole ratio $4: 1$, the electron density profile also extends to about $60 \AA$ in length, consistent with the $\sim 63$ $\AA$ long $\alpha$-helices oriented perpendicular to the plane of the water-gas interface. For the 2:1 mole ratio, the electron density profile extends only about $40 \AA$ with a significantly different overall shape, indicating some substantial distortions in the orientational distribution and/or internal structure of the $\alpha$-helical bundles at the interface due to this higher concentration of chromophores.

3.3. GIXD Studies of Langmuir Monolayers at the Water-Gas Interface. The GIXD data for AP0 and AP0-RuPZn (5:1) Langmuir monolayers for surface area of $100 \AA^{2}$ per $\alpha$-helix are shown as contour plots in Figure 4. The GIXD data show a broad maximum for momentum transfer parallel to the monolayer plane at $q_{x y} \approx 0.6 \AA^{-1}$. This diffraction, which is absent in such data from the aqueous subphase itself, arises due to the interference between parallel helices, which are oriented approximately perpendicular to the water-gas interface. The $q_{x y}$ dependence of the GIXD data from AP0 and AP0-RuPZn (5:1) monolayer and their inverse Fourier transform, namely, the in-plane radial autocorrelation function, were modeled, approximating the helices as solid cylinders of uniform electron density using the analytical expressions developed by Harget and Krimm. ${ }^{47}$ Of course, treating helices as solid cylinders is overly simplistic. Nevertheless, the modeling of the GIXD data was performed to gain some insight into the aggregation state of the peptide bundles. In this regard, several models, describing several possible arrangements of the dihelical form of AP0 (e.g., two-helix bundle, four-helix bundle with four cylinders arranged at the corners of a square, four-helix bundle with four cylinders arranged at the corners of a rhombus, six-helix bundle with six cylinders arranged in the form of a pentagon, and six-helix bundle with six cylinders arranged at the corners of a hexagon) were investigated. These models are shown in Figure S2 in the Supporting Information. The modeling indicates that the dihelices aggregate to form four-helix bundles, which are rotationally disordered about the normal to the membrane plane with glasslike interbundle positional ordering in the monolayer plane.

(46) Strzalka, J.; Liu, J.; Tronin, A.; Churbanova, I. Y.; Johansson, J. S.; Blasie, J. K. Biophys. J. 2009, 96, 4164-4175.

(47) Harget, P. J.; Krimm, S. Acta Crystallogr., Sect. A: Found. Crystallogr. 1971, 27, 586-596. 

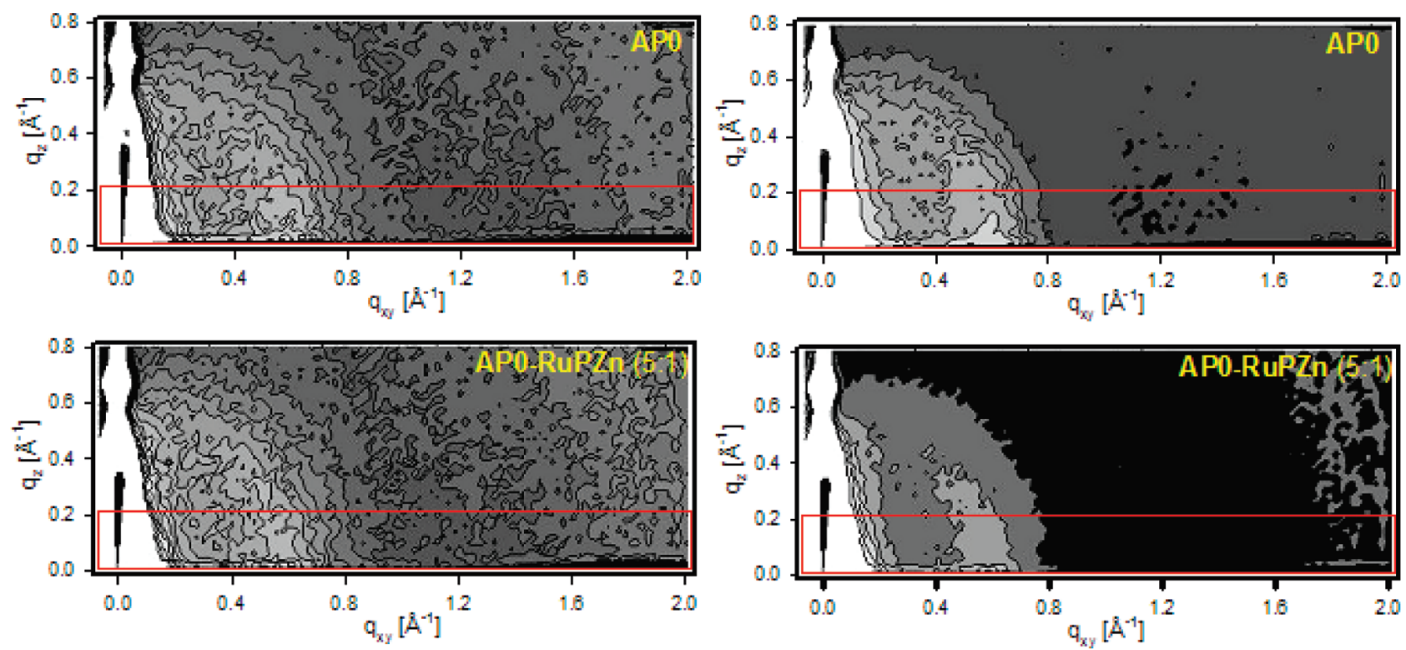

Figure 4. Grazing incidence X-ray diffraction (GIXD) data shown as contour plots for Langmuir monolayers of AP0 and AP0-RuPZn (5:1). The plots on the left and the right sides represent the same data but with different relative scaling to highlight the presence of arc-like interhelical diffraction arising from the orientational distribution of the bundle long axis in both AP0 and AP0-RuPZn (5:1) cases. Data within the red-colored boxes were integrated along the $q_{z}$-direction to provide the average $q_{x y}$ dependence shown in the subsequent Figure 5.

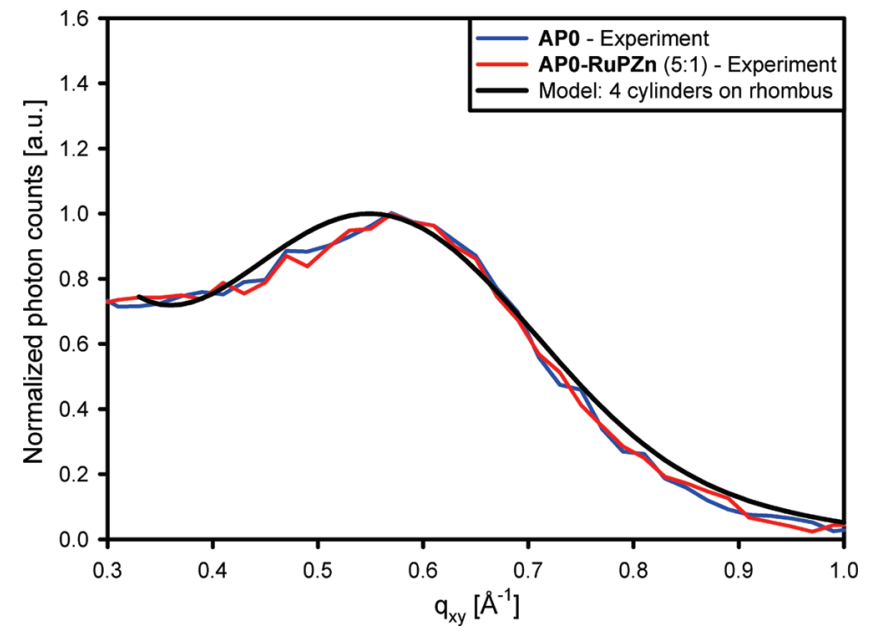

Figure 5. Average $q_{x y}$ dependence of GIXD data from the Langmuir monolayers of AP0 and AP0-RuPZn (5:1) after background subtraction and normalization, shown along with the best fit model (four cylinders arranged at the corners of a rhombus).

Other possible bundles of the AP0 dihelices can be readily excluded since their computed GIXD could not be matched, even qualitatively, with the experimental GIXD data. Comparison of the $q_{x y}$ dependence of the model and experimental GIXD, allowing for the finite $\Delta q_{x y}$ resolution, provides reasonable agreement for a four-helix bundle of $6.0 \AA$ diameter helices at $12.0 \AA$ interhelix separation for a square arrangement (plots not shown) of the four helices in the plane perpendicular to the bundle axis. A rhombic arrangement of the four helices in the plane perpendicular to the bundle axis of the same $6.0 \AA$ diameter, but with $11.85 \AA$ interhelix separation, produces a better fit than a square arrangement. The average $q_{x y}$ dependence of the experimental GIXD data (integrated over the $q_{z}$-range indicated in Figure 4) from both AP0 and AP0-RuPZn (5:1) monolayers are similar and are shown along with the best fit rhombic model in Figure 5 over the range of $q_{x y}$ for which our background subtraction procedure is reliable. The fact that the so-averaged GIXD data arise from the projection of the bundle onto the plane perpendicular to the bundle long axis makes this simplistic model more reasonable.
The GIXD contour plots for the AP0-RuPZn mole ratios of $4: 1$ and $2: 1$, in the spreading solution, are shown in Figure S3 (Supporting Information) and the corresponding average $q_{x y}$ dependence of the experimental GIXD data are shown in Figure S4 (Supporting Information). Comparison of the average $q_{x y}$ dependence of the experimental GIXD data for the mole ratio of 5:1 with those of $4: 1$ and 2:1 indicates some disintegration of the four-helix bundle structure with the increasing chromophore concentration, especially between $4: 1$ and $2: 1$. This result is not surprising given the fact that the insertion of an increasing number of RuPZn chromophores into the interior of the bundle would be expected to diminish the interhelix interactions eventually destabilizing the four-helix bundle structure.

An estimate of the orientation distribution of the peptide bundle axis was determined from the angular distribution of the arc-like interhelix interference in the GIXD data for the AP0 and AP0-RuPZn monolayers. In order to achieve this, the data was transformed from Cartesian coordinates to polar coordinates. The annular region containing the interhelix interference was selected and then integrated radially for each polar angle over an angular range corresponding to the length of the arc. A Gaussian function was fitted via least-squares to this so-derived angular distribution of the radially integrated interhelix interference to obtain a quantitative measure of this estimate of the orientation distribution (i.e., its $\sigma$ value). A representative plot of the angular dependence for an AP0-RuPZn (5:1) monolayer is shown in Figure 6, along with the fitted Gaussian. The $\sigma$ values were found to be about $28^{\circ}$ for a monolayer of AP0 and increasing to about $37^{\circ}$ for all AP0-RuPZn monolayers with the exception of 2:1 mole ratio, for which the angular distribution was essentially constant (i.e., $\sigma$ too large to determine). Such an analysis of the angular dependence of the interhelical interference in the GIXD from the Langmuir monolayers indicates that, while the mean orientation of the four-helix bundle axis is perpendicular to the plane of the water-gas interface, the width of the orientational distribution of the bundle axis over the monolayer ensemble might appear to be quite large, even for the AP0 peptide. However, treating the helices in the bundle as solid cylinders parallel to the bundle axis is a substantial oversimplification and provides only an upper 

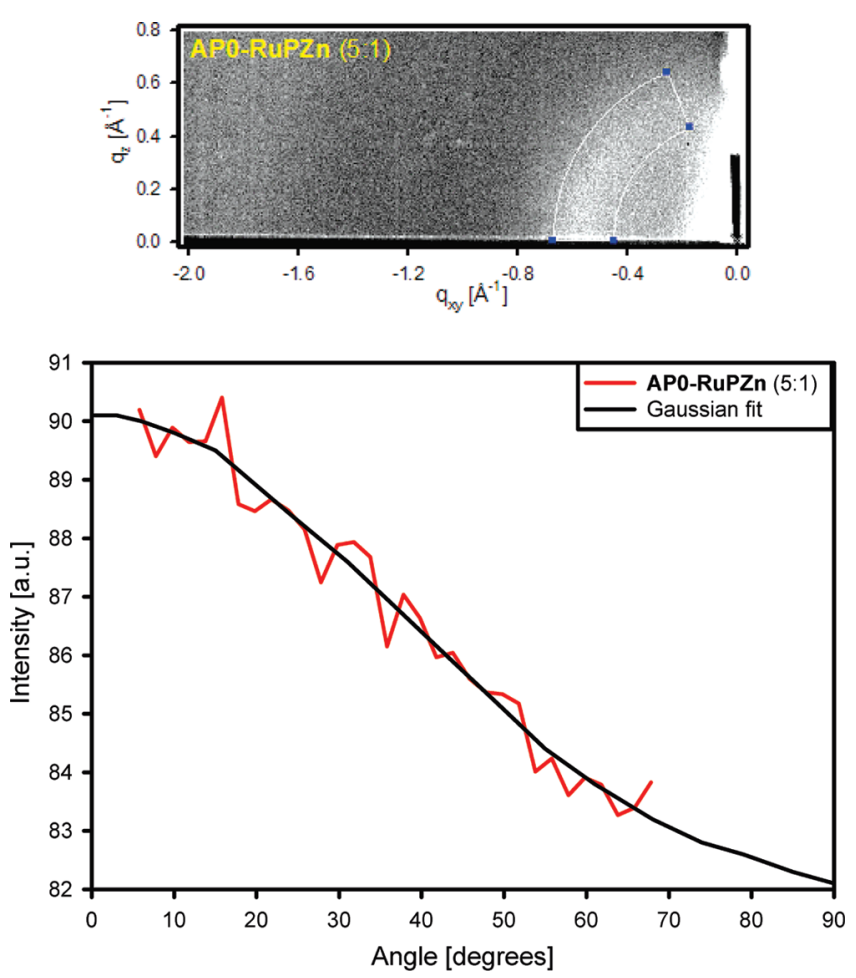

Figure 6. (Upper) GIXD data shown here as a gray scale image for a Langmuir monolayer of AP0-RuPZn (5:1). The annular region (defined by the blue dots/white lines) was integrated radially to produce the average angular dependence of the interhelical diffraction shown as a function of increasing polar angle with respect to the $q_{x y}$ axis and fitted with a Gaussian function (lower), to obtain a quantitative estimate ( $\sigma$ value) of the orientational distribution of the bundle long axis.

estimate for this width. From both experimental GIXD (from Langmuir monolayers) and molecular dynamics computer simulations (of small ensembles at the water-gas interface), it is known that the helices within the bundle already tilt by $\sim 20^{\circ}$ with respect to the bundle axis forming a fairly regular coiledcoil structure over the length of the bundle for AP0.48,38 Incorporation of chromophores into the core of the bundle necessarily induces some variation in the coiled-coil structure over the length of the bundle introducing some range to this tilting of the helices within a bundle. Thus, the actual width of the tilt-angle distribution for the bundle axis itself is very likely to be substantially less than the estimated value by as much as $\sim 20^{\circ}$.

3.4. Linear UV-vis Spectroscopic Investigations on Monolayers on Solid Substrates. The linear UV-vis absorption spectrum measured for AP0-RuPZn monolayer covalently attached to the surface of a fused silica substrate is shown in Figure 7 (after baseline correction). These AP0-RuPZn monolayer specimens on fused silica were prepared in parallel with the corresponding AP0-RuPZn monolayer specimens on the solid silicon substrates for the X-ray reflectivity experiments, as described in the previous section. The spectrum clearly highlights all three characteristic bands of RuPZn chromophore. The fact that the $Q_{x}$-derived band is centered at $656 \mathrm{~nm}$ unequivocally illustrates that RuPZn is bound to the peptide via axial histidyl ligation of the porphyrin $\mathrm{Zn}$ atom. We note that any nonspecifically bound chromophores in this AP0RuPZn monolayer specimen should have been removed during

(48) Zou, H.; Strzalka, J.; Tronin, A.; Blasie, J. K. J. Phys. Chem. B 2007, $111,1823-1833$.

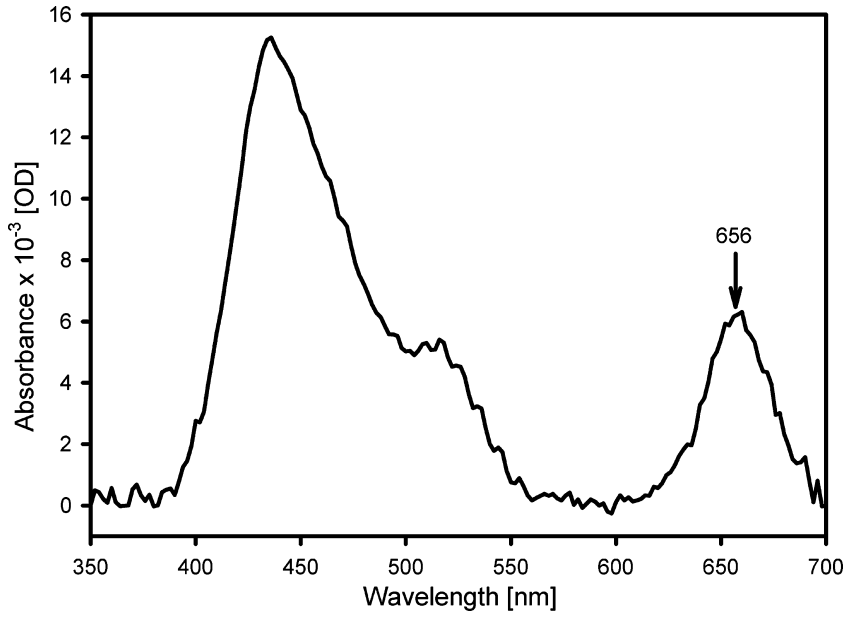

Figure 7. Linear UV-vis absorbance spectrum of AP0-RuPZn monolayer film covalently attached to the surface of a fused silica substrate.

the multiple rinsing steps carried out with ultrapure water. Calculation of the in-plane area per chromophore from the monolayer absorbance yielded an average (over three different monolayers) value $\sim 200 \AA^{2}$ for an area of $100 \AA^{2}$ per $\alpha$-helix, corresponding to an average helix:chromophore mole ratio of $2: 1$. However, we note that the mole ratio of peptide helices to chromophore in any particular covalently attached monolayer could be as high as $1.5: 1$, since the specimens prepared in parallel provided a range of $1.5: 1$ to $2.5: 1$.

3.5. X-ray Reflectivity Investigations of Monolayers on Solid Substrates. The Fresnel normalized X-ray reflectivity data, $R\left(q_{z}\right) / R_{\mathrm{F}}\left(q_{z}\right)$, obtained for the covalently attached monolayers of AP0 and AP0-RuPZn employing the interferometric approach are shown in Figure 8, along with the data from the multilayer reference structure. The added length in the profile structure of the SiNiSi multilayer substrate due to the covalently attached peptide monolayer is manifest in the decreased wavelength of the high frequency fluctuations in the Fresnel normalized reflectivity and not simply in the positions of the major minima, arising from the interference between the SiNiSi multilayer substrate and the peptide overlayer. The electron density profiles obtained, using the box-refinement algorithm with the reference multilayer profile as an additional constraint, for the covalently attached monolayers of AP0 and AP0-RuPZn are shown in Figure 9, along with the profile for the multilayer reference structure.

Based on the fabrication specifications (described in the Experimental Section) employed for the deposition, the features within the various regions (defined by the inequalities noted) of the electron density profile of the multilayer reference structure can likely be assigned to the following layers:

(i) $-150 \AA<z<-90 \AA$; the underlying Si wafer

(ii) $-90 \AA<z<-80 \AA$; native $\mathrm{SiO}_{2}$ initially on the wafer surface prior to deposition

(iii) $-80 \AA<z<-40 \AA$; first amorphous Si layer deposited on the native oxide layer

(iv) $-40 \AA<-15 \AA$; amorphous Ni layer deposited on the amorphous $\mathrm{Si}$ layer

(v) $-15 \AA<z<0 \AA$; ; second amorphous Si layer deposited on the Ni layer

(vi) $0 \AA<z<+20 \AA$; low-density amorphous $\mathrm{SiO}_{x}$ layer on the substrate's surface

Although the amorphous $\mathrm{Ni}$ layer and $\mathrm{Si}$ layers were deposited separately following the fabrication specifications, not 

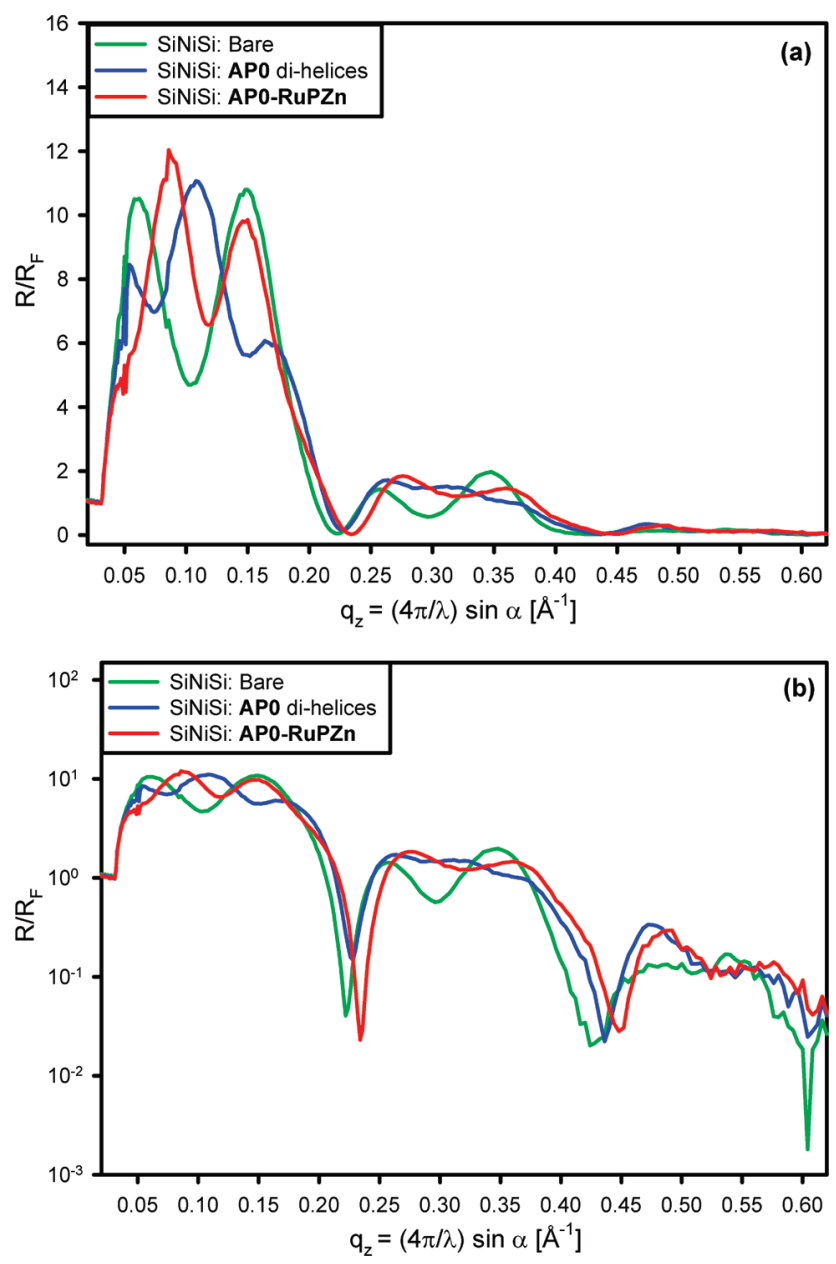

Figure 8. Fresnel normalized X-ray reflectivity for AP0 and AP0-RuPZn monolayer films covalently attached to the surface of a SiNiSi multilayer structure on silicon ((a) linear ordinate scale and (b) log ordinate scale). The X-ray reflectivity from the SiNiSi multilayer structure on silicon itself, utilized as the reference structure in the interferometric phasing procedure, is also shown in both.

unexpectedly, some interdiffusion of $\mathrm{Ni}$ into underlying amorphous silicon layer seems apparent with some accumulation at the silicon-amorphous silicon oxide interface, as indicated by the electron density profile features in this region. Otherwise, the multilayer substrates are well-suited to the interferometric approach for the investigation of the profile structures of the peptide overlayers subsequently covalently attached onto the amorphous $\mathrm{SiO}_{x}$ surface. Hence, all subsequent discussion will focus only on the covalently attached peptide overlayers.

A substantial improvement in both the spatial resolution and sensitivity arising from the interference effects is clearly evident from the comparison of Figure 8 with Figure 3 . The signal from the peptide monolayer in the interferometric case is contained in the difference between the Fresnel normalized reflectivity for the reference structure itself and that with the covalently attached monolayer. This relatively large amplitude signal is virtually noiseless, extending over the entire range of $q_{z}$ investigated, especially evident on a semilog scale. In this case, the spatial resolution achievable in electron density profile of the peptide monolayer derived from these data is limited only by the range of momentum transfer investigated. Fourier transformation of the Fresnel normalized data provides the autocorrelation of the gradient of the electron density profile of the reference multilayer structure (Patterson function), which
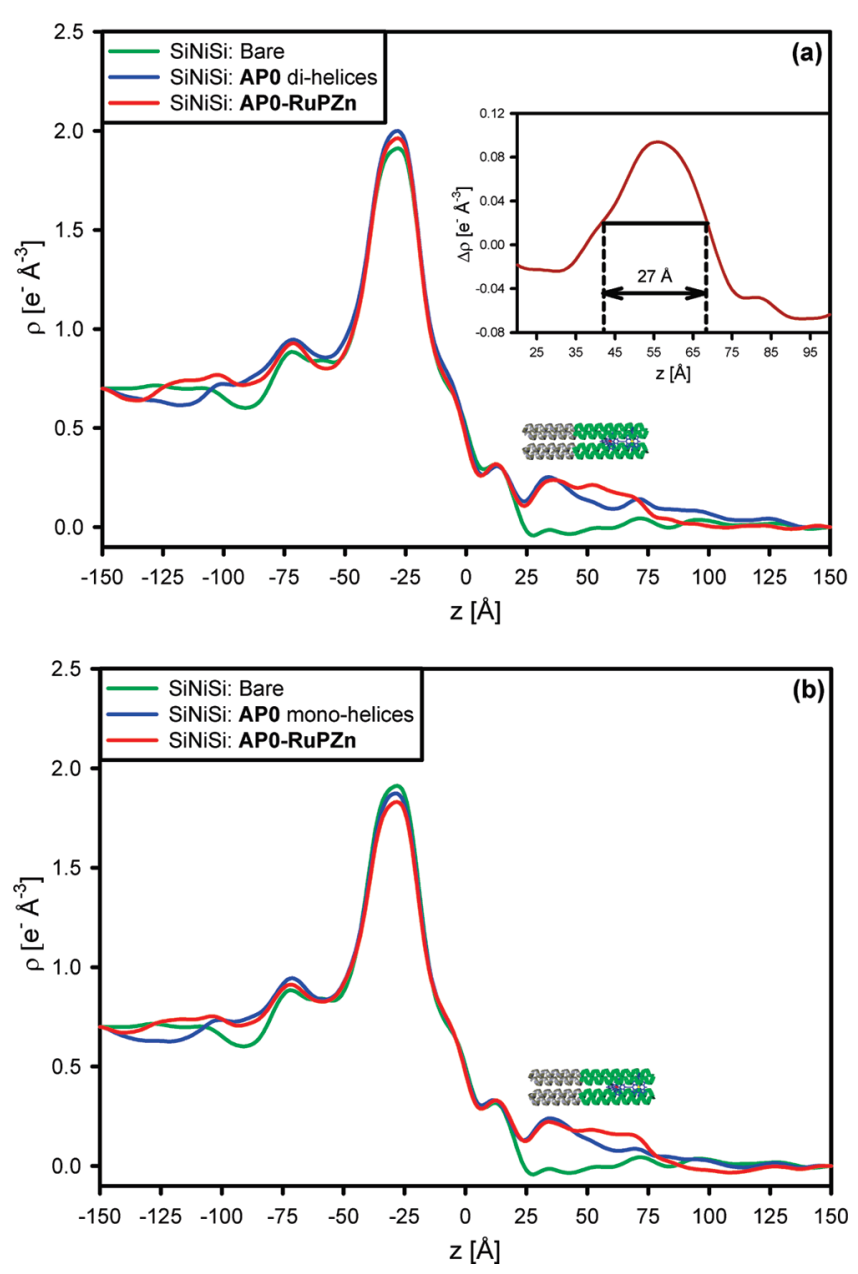

Figure 9. (a) Electron density profiles for monolayer films of AP0 and AP0-RuPZn covalently attached to the surface of a SiNiSi multilayer structure on silicon, together with that for the SiNiSi multilayer structure on silicon itself. Schematic representation of the AP0-RuPZn is shown above approximately to scale. The N-terminal cysteine residues were covalently linked via a disulfide bond to form dihelices prior to their exposure to the linker at the alkylated surface of the inorganic substrate in this case. Inset: Difference electron density between AP0-RuPZn and AP0 monolayer films, shown on an expanded scale. (b) Electron density profiles for monolayer films of AP0 and AP0-RuPZn covalently attached to the surface of a SiNiSi multilayer structure on silicon, together with that for the SiNiSi multilayer structure on silicon itself. Schematic representation of the AP0-RuPZn is shown above approximately to scale. However in this case, the N-terminal cysteine residues were not covalently linked via a disulfide bond prior to their exposure to the linker at the alkylated surface of the inorganic substrate in this case.

is of finite extent. The significant features in this autocorrelation extend to appropriately larger distances by $\sim 60 \AA$ when the peptide monolayer is covalently attached to the multilayer substrate's surface (plots not shown). In the electron density profile itself, the peptide-substrate interface is at about $+20 \AA$ and the peptide-air interface is at about $+80 \AA$ for both AP0 and AP0-RuPZn monolayers, illustrating that the peptide extends to $\sim 60 \AA$ in length, consistent with the increased extent of the autocorrelation function mentioned above. The average electron density of the AP0 monolayer is about $0.25 \mathrm{e}^{-} \AA^{-3}$, which is less than the expected value of $0.38 \mathrm{e}^{-} \AA^{-3}$ for the fully hydrated peptide, the electron density decreasing with extension from the substrate surface. A majority of the decreased average electron density is likely due to the decreased hydration of the peptide bundle in the moist helium environment. We note that there is also the possibility of some small degree of 
incomplete surface coverage following removal of any noncovalently attached peptides, thereby allowing the remaining bundles to tilt with respect to the surface normal introducing some disordering with distance from the surface. Upon incorporation of RuPZn chromophore, the electron density of AP0RuPZn monolayer is more uniform over the length of the bundle and substantially higher than the AP0 monolayer, particularly in the region between $+42 \AA$ and $+69 \AA$. This suggests that the greatest perturbation of the peptide bundle upon chromophore incorporation occurs over this region, arising from changes due to the chromophore electron density itself as well as changes in the local peptide conformation and hydration. The difference electron density profile for AP0-RuPZn-minus-AP0 is shown on an expanded scale in the insert in Figure 9. The full-width at half-maximum (fwhm) of the features characterizing this perturbation is $\sim 27 \AA$, again comparable to the expected length of $24 \AA$ for the RuPZn chromophore. Both the position and width of these features are again consistent with the expected localization of the RuPZn chromophore along the length of the peptide bundle, as shown in the schematic representation of the AP0-RuPZn peptide-chromophore complex in Scheme 1. The amplitude of the features characterizing this perturbation is substantially larger here for the covalently attached monolayers because the helix to chromophore stoichiometry in the monolayer is $\sim 2: 1$, possibly as high as $1.5: 1$ based on the in situ spectroscopy of the parallel specimens, with a much lesser contribution from water hydrating the AP0-RuPZn complex than for the Langmuir monolayer of the AP0-RuPZn. Lastly, we note that the monolayer electron density profiles (Figure 9) are virtually identical (both for the AP0 and AP0RuPZn), irrespective of whether the individual helices are covalently linked via a disulfide bond bridging their N-terminal cysteine residues to form dihelices prior to their exposure to the linker at the alkylated surface of the inorganic substrate. This indicates that the thioether bond between the linker and the N-terminal cysteine side chain is stronger than the interhelix disulfide bond.

\section{Conclusions}

We have developed a strategy to effectively control both the orientational and positional ordering, and thereby the macroscopic NLO response, of a highly hyperpolarizable RuPZn chromophore in monolayer ensembles, using the robust synthetic amphiphilic four-helix bundle peptide AP0 as a host. In this article, we have characterized the system from the precursors to the final material employing both X-ray techniques and in situ linear UV-vis absorption spectroscopy. The structural studies performed on Langmuir monolayers at the water-gas interface indicate that the amphiphilic four-helix bundle AP0 peptide can vectorially orient the extended, conjugated RuPZn chromophore in peptide-chromophore ensembles on the macroscopic length scale at stoichiometries as high as one chromophore per five helices, i.e., approximately one chromophore per four-helix bundle. The vectorial orientation is achieved via the specific axial histidyl ligation of the porphyrin-coordinated $\mathrm{Zn}$ ion of the RuPZn chromophore in the nonpolar core of the bundle, coupled with the strong amphiphilicity of the bundle exterior. The location of the RuPZn chromophore along the length of the peptide bundle was also determined. A viable "directed-assembly" method for the covalent attachment of monolayer ensembles of the AP0 peptide to the surface of a solid inorganic substrate has been presented. Incubation of these covalently attached monolayers with the RuPZn chromophore followed by washing resulted in stoichiometries as high as one chromophore per dihelix in the resulting monolayers of AP0RuPZn. The structures of these covalently attached ensembles have similarly been described. In particular, the location of the RuPZn chromophore along the length of the peptide bundle determined from the Langmuir monolayers was confirmed, facilitated by the higher stoichiometries achieved with the covalently attached monolayers. This work provides the essential basis for characterizing the nonlinear optical response of these monolayer ensembles that firmly establishes the unique vectorial orientation of the RuPZn chromophore throughout the monolayer ensemble, as described in the accompanying article.

Acknowledgment. This work was supported primarily by a grant from the Department of Energy Biomolecular Materials program DE-FG02-04ER46156 (V.K., M.J.T., and J.K.B.). Additional partial support was provided by the National Science Foundation MRSEC program DMR05-20020 and NSEC program DMR-0425780 (A.T. and J.S.). We thank Jaseung Koo and Jing Liu for assistance with X-ray data collection. We acknowledge Ivan Kuzmenko and Thomas Gog of APS for their assistance in the use of the liquid surface spectrometer. We thank Paul Heiney for providing a free license for the Datasqueeze software, Chian Liu of APS for fabrication of multilayer reference structures on the $\mathrm{Si}$ wafer, and Ali Khounsary of APS for design and manufacture of the GIXD beamstop. Use of the Advanced Photon Source was supported by the U.S. Department of Energy, Office of Science, Office of Basic Energy Sciences, under Contract No. DE-AC02$06 \mathrm{CH} 11357$.

Supporting Information Available: (1) Fresnel-normalized $\mathrm{X}$-ray reflectivity data and electron density profiles for Langmuir monolayers of AP0-RuPZn (at helix to chromophore mole ratios 4:1 and 2:1). (2) Different cylinder models investigated for fitting the GIXD data. (3) Grazing incidence X-ray diffraction (GIXD) data shown as contour plots for Langmuir monolayers of AP0-RuPZn (helix to chromophore mole ratios 4:1 and 2:1). (4) Average $q_{x y}$ dependence of GIXD data from the Langmuir monolayers of AP0-RuPZn (at helix to chromophore mole ratios 4:1 and 2:1). This material is available free of charge via the Internet at http://pubs.acs.org.

JA1010702 